\title{
El arte por el dolor: resemantización estética de la crueldad en "The Birthday of the Infanta" de Oscar Wilde
}

\author{
Eduardo Valls Oyarzun \\ Complutense University, Spain
}

Copyright (c) 2020 by Eduardo Valls Oyarzun. This text may be archived and redistributed both in electronic form and in hard copy, provided that the author and journal are properly cited and no fee is charged for access.

\begin{abstract}
Oscar Wilde's fairy tale "The Birthday of the Infanta" (1891) echoes an ellective affinity, which hitherto has not been discuss very often, between Friedrich Nietzsche's philosophy and Oscar Wilde's aesthetic principles, to wit, the idea of cruelty, its association with pleasure and its depiction in Art and Culture. The essay discusses these ideas in the tale, by pinpointing the specific affinities between both authors, which are semiotic and aesthetic in nature. Thus, the essay boasts a novel interpretation of the tale, firstly, because it highlights the ideas of reading and interpretation of cruelty in an aesthetic milieu as the textual driving force; secondly, because the analysis categorically challenges traditional moral readings of the story, which nowadays still inform a great deal of critical readings of the text.
\end{abstract}

Key Words. Oscar Wilde, Friedrich Nietzsche, Decadent Movement, Cruelty, aestheticism.

Resumen. El relato "The Birthday of the Infanta" (1891), de Oscar Wilde, refleja una de las afinidades electivas menos exploradas entre el pensamiento del filósofo alemán Friedrich Nietzsche y los principios estéticos del autor irlandés, en concreto: el concepto de crueldad, su asociación con el placer y la sublimación de esta idea en el arte y la cultura. Se exploran estos principios en el relato, señalando las coincidencias de planteamiento entre ambos autores, que son de carácter semiótico y estético. Así, se da un nuevo sentido al relato, primero, porque se incorpora la noción de lectura e interpretación de la estética de la crueldad como principio rector del texto, y, segundo, porque el análisis permite rechazar categóricamente la lectura moral tradicional que, aun hoy, impregna buena parte de las lecturas críticas del relato.

Palabras clave. Oscar Wilde, Friedrich Nietzsche, decadentismo, crueldad, esteticismo. 


\section{Introducción}

Se presenta aquí una nueva lectura del cuento infantil de Oscar Wilde (1854-1900), "The Birthday of the Infanta" ("El cumpleaños de la Infanta"), aparecido en su segunda colección de cuentos infantiles, A House of Pomegranates (1891). Esta nueva lectura se sostiene sobre la idea común que acerca del dolor y la crueldad, así como su expresión artística y cultural, compartían el autor irlandés y el filósofo alemán Friedrich Nietzsche (1844-1900). Esta afinidad concreta y específica (el valor del dolor y la crueldad) en el pensamiento de ambos autores apenas si ha sido señalada en muy contadas ocasiones. Thomas Mann (170), el primer autor que comenzó a ver afinidades entre ambos autores, ni siquiera considera similitud alguna entre el pensamiento de ambos al respecto del instinto de crueldad; Patrick Bridgewater indica que "Wilde's and Nietzsche's ideas correspond ... on a range of issues" (366), entre los que incluye, sin más explicación ni mayor desarrollo, la idea del dolor; Eduardo Valls Oyarzun sugiere la afinidad en relación con las ideas que ambos autores sostenían sobre el cristianismo (215), mientras que Kate Hext ("Rebels" 202-220) y Julia Prewitt Brown (67-105), al igual que Mann, obvian la afinidad por completo.

Las veces que esta idea ha sido señalada como espacio de solapamiento intelectual entre Wilde y Nietzsche, en resumen, son escasas, poco extensas y en modo alguno sistemáticas. Sin embargo, "The Birthday of the Infanta" constituye un ejemplo paradigmático de cómo Wilde asocia la idea de crueldad a la noción de placer en sentido semiótico y cultural, al modo como lo analiza también Nietzsche en Anticristo (2016), La genealogía de la moral (2016) y Más allá del bien y del mal (2016). El desarrollo del concepto de crueldad en sus diversas manifestaciones, siempre ligado a la idea de placer, servirá como ángulo crítico desde el que enfocar un conflicto fundamental del relato que tampoco ha sido discutido en profundidad. Se trata, en concreto, de la incapacidad de leer los códigos culturales que ordenan, administran y canalizan tanto el dolor como la crueldad y el sufrimiento en tanto que fenómenos insoslayables de la experiencia vital, para entender, al fin y al cabo, que el relato añade un matiz de orden político tanto a la idea amoral del arte como a la concepción estética del mundo que define el discurso crítico de Oscar Wilde.

\section{Cruel hermosura; hermosa crueldad}

La estética que conforma "The Birthday of the Infanta" exhibe un claroscuro general que surge del contraste entre la belleza sensual (las manifestaciones artísticas que abundan en el relato) y la atmósfera de crueldad, dolor y sufrimiento que se articula en su subtexto. Cualquier aspecto del relato parece remitir a una manera de violentar la realidad, retorciendo su sentido tradicional y poniendo de manifiesto, en consecuencia, las expresiones de dolor que se derivan de dicha violencia. Los personajes de la corte de España, sin embargo, parecen convivir bien con esta estructura semiótica del dolor, y hasta muestran distintas formas de integrarla en el espacio sociocultural que habitan. Hay cuatro personajes principales en el cuento: tres de ellos son habitantes de la corte: Don Pedro de Aragón, el Rey de España (hermano de Don Pedro) y la propia Infanta. El cuarto personaje, el Enano, es precisamente quien altera el raro equilibrio cruel que domina el espacio palaciego. Un grupo de personajes secundarios que incluye al Gran Inquisidor de Granada y al Chambelán completa el elenco. Todos ellos, personajes principales y secundarios, comparten un rasgo común de crueldad que preside la vida en la corte, aunque la manifestación de dicho rasgo resulte ser distinta en cada caso particular.

Don Pedro de Aragón se describe explícitamente como el personaje más cruel de todos - "his cruelty, even in Spain, was notorious" (Wilde, "Infanta" 99) -. Su naturaleza intrigante (es sospechoso de haber matado a la reina) transparenta un cambio sustancial en la estructura política de la corte, una dislocación - se entiende que ilegítima - del centro de poder, al actuar 
el personaje de facto como cabeza del Estado en sustitución del Rey. La autoridad de su gobierno proviene del terror que infunde: "When they caught sight of Don Pedro they scowled at him, and some of them looked terrified, for only a few weeks before he had had two of their tribe hanged for sorcery in the market-place at Seville" (Wilde, "Infanta" 103).

El Rey difiere de Don Pedro en su forma de canalizar la crueldad. El personaje mantiene una actitud en apariencia ambigua hacia el sufrimiento, la cual, irónicamente, oculta el disfrute hedonista que surge de poner en práctica el instinto de crueldad. Su amor por la Infanta y su odio por Don Pedro calificarían en principio al personaje como figura de carácter templado y cariñoso (Wilde, "Infanta" 98), pero el Rey puede llegar a comportarse de forma tanto o más cruel que el propio Don Pedro. El gusto del monarca por los "autos de fe" - celebra su boda con uno "especialmente solemne" (Wilde, "Infanta" 98) - revela su capacidad para disfrutar del sufrimiento ajeno antes de la muerte de la reina. Tras esta, la forma preferida del Rey para ejercer su instinto de crueldad se comprende mejor al amparo de las ideas de Nietzsche con relación a la administración de la crueldad en la cultura occidental. El rasgo que mejor define al Rey es la melancolía. Tras la muerte de la reina, el monarca se sume en una profunda crisis de tristeza. A fin de paliar dicha crisis, el Rey se las arregla para embalsamar el cadáver con la ayuda de un "Moro" que ha sido "condenado por el Santo oficio" (Wilde, "Infanta" 98). Más tarde, quizá intentando ocultar la transgresión a las autoridades religiosas, el Rey esconde en secreto el cuerpo de la reina en una "capilla negra" (Wilde, "Infanta" 98). Así las cosas:

Once every month the King, wrapped in a dark cloak and with a muffled lantern in his hand, went in and knelt by her side, calling out, 'Mi reina! Mi reina!' and sometimes breaking through the formal etiquette that in Spain governs every separate action of life, and sets limits even to the sorrow of a King, he would clutch at the pale jewelled hands in a wild agony of grief, and try to wake by his mad kisses the cold painted face. (Wilde, "Infanta" 99)

La cadencia temporal, el escenario fantasmal y el atuendo de la capa oscura ayudan a poner en marcha un rito abiertamente subversivo contra los usos culturales (la moral colectiva) de Palacio al objeto de celebrar el cadáver de la reina. El erotismo implícito en el cuerpo sin vida permite al rey liberar su sentido hedonista durante las frecuentes visitas que realiza a la capilla, pero no porque la relación háptica sobre la que pone el foco el pasaje revele un placer libidinoso más o menos perverso, sino porque dicha relación ayuda a localizar el origen y destino de ese placer concreto en la agonía que propicia la tristeza. El Rey llega a articular la relación entre placer, dolor (en forma de melancolía agónica) y belleza de manera explícita, sustituyendo el deseo erótico por el deseo del dolor: "he bade the ambassadors tell their master that the King of Spain was already wedded to Sorrow, and that though she was but a barren bride he loved her better than Beauty" (Wilde, "Infanta" 99). El origen de la actitud del Rey se localiza en una poderosa y placentera introyección de su instinto de crueldad a la manera como la entiende Friedrich Nietzsche. La devoción del monarca hacia una práctica ritual de tonos tan hedonistas, así como su vinculación con la idea de tristeza como tortura sugieren un sentimiento placentero de mortificación que nace en el gozo derivado de generar dolor, de poner en práctica "la estructura de la vida como vida activa" manifestada "en ver sufrir o incluso en infligir el sufrimiento" (Deleuze 183). El instinto cruel del monarca es patente, pero no se manifiesta hacia fuera, como en el caso de Don Pedro, sino hacia dentro, hacia el propio sujeto:

Claro está, hay que expulsar a la torpe psicología de otros tiempos, que sobre la crueldad solo supo enseñar que surgía en la visión del sufrimiento ajeno: también hay un goce abundante, sobreabundante en el propio sufrimiento, en el hacerse-sufrir-a-símismo. (Nietzsche, Más allá 392) 
Nietzsche sitúa la introyección de la crueldad, producto del devenir de las ideas morales en el contexto civilizador de la cultura, en el origen de la mala conciencia (tema del segundo tratado de La genealogía de la moral), y desarrolla la idea en el contexto de su visión del hombre moderno como individuo convertido "en herida dolorosa", en dislocación entre "lo que en él piensa" y "lo que en él vive" (Safranski 323). Esta dualidad - ique no dialéctica! (Deleuze 195) - entre la afirmación vitalista del instinto del cuerpo y su contrapartida en forma de "espíritu que poda la vida" se construye a través de un espacio semiótico forjado en "la constancia", en una "conducta calculable", al "tejer una red de rituales y formas de comportamiento" con las que se "inserta una conciencia en el instinto" (Safranski 323-324). La estructura psicofisiológica del planteamiento nietzscheano denota que el instinto de provocar sufrimiento y dolor no se ha suprimido, sino que se ha domado, a veces incluso para ser utilizado como arma ideológica. Esto se ha logrado con una herramienta muy específica, la conciencia, creada para articular el espacio inter subjetivo, esto es, la civilización y la cultura.

El proceso por el que el monarca reconfigura como ritual su disfrute del placer, proyectando de facto hacia el interior el instinto que previamente se permitía a sí mismo disfrutar de manera exotérica, revela lo que Kate Hext denominaría "a pertinent and specific "elective affinity"” ("Rebels" 205) entre el relato de Wilde y la propuesta psico-fisiológica de Nietzsche, que no puede pasarse por alto en un contexto revisionista de la moral como el que exploran tanto el filósofo alemán como el autor irlandés. La propia Hext, sin embargo, no termina de identificar esta "afinidad electiva" entre autor y filósofo, aunque sí provee de un marco teórico para explorarla, al menos parcialmente.

La puesta en escena del sufrimiento por parte del Rey, es decir, el elemento ritualístico que define su gozo del dolor, transparenta una concepción estética del yo que resulta ser común a ambos autores:

Wilde and Nietzsche are brought together by their shared pursuit of what I have, above, called Romantic Individualism. ... In common with German Individualism, Romantic Individualism defines the individual according to his creativity, 'uniqueness, originality, self-realisation'. Romantic Individualism, then, like German Individualism has 'relf-realisation' at its centre, founded on the belief that the individual is unique, and unbounded by external conditions and limitations. This 'self-realisation' is achieved through aesthetic experience, through which the individual may create him or herself. (Hext, "Rebels" 205)

Hext concibe el "individualismo romántico" exclusivamente en términos de creación artística, de transgresión estética entendida "as a response against the 'Victorian Individualism' of nineteenth-century Britain" ("Rebels" 206). Hext comprende, por tanto, que una construcción artística del yo fundada sobre todo en la trascendencia de las categorías morales tradicionales implica el mayor acto de disidencia contra el "Victorian Individualism that un-Romantically links identity to politics and economics" ("Rebels" 206) por parte de Wilde y Nietzsche.

Hext no advierte, en cambio, la capacidad transgresora implícita en la introyección de la crueldad que Nietzsche teoriza y Wilde pone en práctica en el personaje del Rey. La reivindicación del dolor como fuente de gozo solipsista (señalada en el Rey a través de la transferencia entre deseo erótico y angustia) implica trascender el uso ideológico y normativo que la cultura ha venido haciendo de la introyección de la crueldad. La cultura se vale de este fenómeno psico-fisiológico para volver el espacio inter subjetivo arriba mencionado contra el individuo que no cumple con el precepto de la norma, la moral divina o la ley, dando pie en consecuencia a la "culpa" o "mala conciencia" (Nietzsche, Genealogía 470-472). La reivindicación del dolor como placer en el caso del Rey supone todo lo contrario; implica 
superar el uso normativo y social del fenómeno, al tiempo que subraya el potencial individualista implícito en la "singularidad" privada, única y exclusiva de la experiencia física del dolor, es decir, del placer. Es razonable afirmar, por tanto, que esta particular "afinidad electiva" entre Nietzsche y Wilde (la introyección de la crueldad) podría coadyuvar a realizar el "Romantic Individualism" que propone Hext.

La introyección de la crueldad no se trata de la única afinidad electiva que exhibe el relato de Wilde con el pensamiento de Nietzsche. Como ya se ha sugerido y ahora se desarrollará, Nietzsche también explora los modos en que la cultura ha logrado construir una semiótica cultural del dolor.

\section{Hacia una semiótica del dolor}

El personaje de la Infanta acaso resulte la figura más compleja del relato. Percibe el monarca en su hija "her perecocious sex appeal" (Pendlebury 131), asociado al recuerdo de su esposa muerta, y, por ende, a la "terrible agony of [his married life's] ending" (Wilde, "Infanta" 99). En la niña confluye una serie de atributos que evocan dolor y sufrimiento, como si la propia Infanta se erigiera en signo o símbolo modular de la crueldad, pero sin sufrir "no pains of conscience", "[no] moral investment" que pudieran alterar su inocencia estética (Jones 887888). La circunstancia resulta particularmente significativa durante la celebración del espectáculo de variedades organizado para su cumpleaños, una serie de "circus-type performances suggesting a Hadean freak show" (Pendlebury 131). Allí, la respuesta de goce y placer con que la Infanta y sus amigos saludan las pequeñas manifestaciones de "realidad diluida" (Jones 888) contenidas en las actuaciones completa una estructura estética por causa de la cual los niños (y la Infanta notablemente sobre ellos) condicionan la representación del dolor al valor fundamental de la belleza pura.

Los distintos números de variedades que conforman la fiesta de cumpleaños combinan siempre dos características concretas: crueldad y juego. Hay una "sham-bullfight", que la Infanta considera "much nicer ..., than the real bull-fight that she had been brought to see at Seville" (Wilde Infanta 100). También hay una representación de "the semi-classical tragedy of Sophonisba" (Wilde, "Infanta" 101) tras la cual, la Infanta llora desconsolada. El siguiente número consiste en una danza que a su vez evoca un curioso episodio acerca de un intento de envenenamiento (Wilde, "Infanta" 102-103), lo cual deja sin palabras, fascinada, a la Infanta. Tras esto, un grupo de gitanos canta y baila para ella. Irónicamente, los bailarines piensan que "one so lovely as she was could never be cruel to anybody" (Wilde, "Infanta" 103). Son estos mismos bailarines quienes luego ponen en escena un numerito de animales amaestrados: bajo la atenta mirada de un oso cabeza abajo, unos "wizened apes" escenifican una pequeña batalla campal y unas maniobras militares (Wilde, "Infanta" 103), tras lo cual llega la actuación del Enano deforme, el personaje que domina en la segunda parte del relato y cuya exhibición cierra el espectáculo de variedades.

El catálogo de actuaciones de la fiesta no es muy distinto del que cita Nietzsche en Más allá del bien y del mal y La genealogía de la moral como manifestaciones culturales del instinto de crueldad sublimado. En ambos textos el filósofo presenta una relación de fenómenos culturales tras los cuales subyace la necesidad de ventilar el deseo por causar y ver causar dolor en un contexto de control colectivo: desde la brutalidad hacia los animales en general - "lo que el romano saborea en la arena" - y "las corridas de toros" en particular hasta "la dolorosa voluptuosidad de la tragedia" (Nietzsche, Más allá 389-390), pasando por "ejecuciones, suplicios" o "autos de fe" (Nietzsche, Genealogía 490) - también presentes en la fiesta del relato de Wilde $-\mathrm{y}$, notablemente, la humillación al bufón en la corte (como sucede, también, en el relato de Wilde): "En cualquier caso, no hace todavía mucho tiempo [era impensable] ... una casa noble en que no hubiese alguien en que poder descargar sin escrúpulos la maldad y las 
burlas crueles" (Nietzsche, Genealogía 490). Nietzsche ilustra con estos ejemplos como "casi todo lo que llamamos "cultura superior" descansa sobre una espiritualización y profundización de la crueldad" (Nietzsche, Genealogía 491), dado que "el dolor no es un argumento contra la vida, sino al contrario, un excitante de la vida, un encanto para la vida, un argumento a su favor" (Deleuze 183). Adelantando los principios que Freud sostiene en El malestar en la cultura, aunque desde una perspectiva psico-fisiológica, Nietzsche caracteriza el valor estético de la ficción y la cultura como herramientas principales de civilización a la hora de encauzar y administrar, a través de la representación, los impulsos hedonistas implícitos en el instinto de crueldad: "aquella bestia salvaje no ha muerto en absoluto", sostiene Nietzsche (Más allá 390), sino que se ha domado en la ficción del arte y la representación. Nietzsche establece de este modo, además, que "art preceded morality, and transcends it, or, rather, art should define morality" (Allen 395), recontextualizando así el valor del dolor, cuyo marco de significado debe pasar de la moral a la estética. Y este es el mismo sentido en que Wilde, mutatis mutandis, comprende el fenómeno estético, a saber, como una forma de encauzar, de dormar, precisamente, los peligros de la existencia: "It is through Art, and through Art only, that we can realise our perfection; through Art, and through Art only, that we can shield ourselves from the sordid perils of actual existence" ("Critic" 174).

Este planteamiento se presta a una doble vertiente de análisis. De una parte, Nietzsche subraya el valor del placer propiciado en el acto de infligir dolor; de otra, el filósofo identifica la administración del dolor en la "cultura superior" (Más allá 391) como semiótica artística, un juego de reglas ficticio por y a través del cual se articula la experiencia.

En este sentido, el lector de "The Birthday of the Infanta" no debería pasar por alto que Wilde concibe la crueldad a la manera más puramente nietzscheana, de forma lúdica, diríase incluso juguetona. El elemento del juego resulta insoslayable. La corrida de toros no es real, sino de pega. La tragedia - acaso la más alta sublimación de la crueldad para Nietzsche - no es más que un guiñol; la evocación del intento de envenenamiento se realiza a través de la danza; el sometimiento al animal se lleva a cabo en un espacio lúdico-deportivo, etc. Hay un sentido del juego y lo lúdico en general que resulta sugerente en el contexto de cuento infantil que enmarca el relato. El juego proyecta un sentido de orden y reglas, a través de las cuales la comunidad de individuos se somete a una estructura semiótica por causa de la cual se refiere el significado del propio juego. Solo mediante el acuerdo entre jugadores se consigue establecer la funcionalidad del juego, de modo que la imposibilidad de comprender las reglas coloca en cualquier caso al jugador fuera del contexto semiótico del juego. Ahora bien, esto es tan cierto para la estructura del juego como para el planteamiento de la estructura cultural que predomina en el relato. En cierto sentido, la Infanta y sus compañeros celebran la crueldad única y exclusivamente porque esta se da en forma lúdica. Sostiene Jones que esta es una forma de diluir el valor de la experiencia humana (888), pero lo cierto es que la vivencia del placer cruel se produce porque el instinto de la crueldad se da a priori en los personajes, especialmente en el de la Infanta; y porque tal instinto se canaliza a través de la estructura cultural del juego como patrón ordenador de la experiencia vital, no como elemento que diluya dicha experiencia. De este modo se sugiere un tipo de sinécdoque entre el juego y la alta cultura, vinculados entre sí por su naturaleza común de sistemas semióticos, pero también por su función de administrar, canalizar y reevaluar el instinto de crueldad.

Desconocer las reglas del juego implica confundir los niveles de ficción y realidad, y esto puede resultar fatal para la construcción e interpretación del sistema estético. La crueldad debe ejercerse en el nivel adecuado de la ficción del juego, que es lo que ocurre en el relato, por cuanto "the artifice preserves the uninhibited enjoyment of a symbolically violent performance" (Jones 889); pero esto no significa que el juego sea ajeno a la experiencia de la vida, que de facto se traslada al espacio lúdico de la ficción en contraposición a la realidad. El juego y la ficción que impone resultan ser el marco sémico cultural que genera los valores sobre 
la crueldad. No mantener la pretensión ficticia del juego, de la cultura, supone en última instancia introducir un elemento perturbador, ajeno al acuerdo comunitario y civilizador que se propicia en la representación estética del dolor, y que se arroga la aplicación ética de la crueldad, bien en forma de castigo - ejercicio de la crueldad exotérica, por parte del Estado - o bien en forma de culpa - ejercicio de la crueldad de forma esotérica, por parte del propio Individuo (Nietzsche, Genealogía 470-472) -. Ambos autores, Wilde y Nietzsche, comparten en el fondo una condición de estetas que se manifiesta en la comprensión del arte como espacio para articular la experiencia humana como elemento redentor de la vida.

\section{Tragedia en miniatura}

Tal y como sugiere John Albert, "The Birthday of the Infanta" constituye una fábula sobre las vicisitudes propias de animales y objetos inanimados (245). El texto se deleita en la descripción de texturas, colores, formas, y todo tipo de estímulos sensuales. La dimensión estética del relato se lleva a cabo en el acento físico - corpóreo, dionisiaco - de la narración, en la estimulación sensorial más pura, que apela a la interpretación del mundo desde una perspectiva fisiológica, ajena al espacio cultural prescrito por el discurso normativo victoriano. Así, Wilde refiere "hypnotic, intensely aesthetic experiences simply to imagine color, taste, smell, and sight in a vastly different world from the English nursery" (Wood 166), proyectando en la escenografía del texto un significado por causa del cual logra canalizarse culturalmente la administración del dolor, el sufrimiento y la crueldad. Tómese, a modo de ejemplo, el siguiente pasaje:

The purple butterflies fluttered about with gold dust on their wings, visiting each flower in turn; the little lizards crept out of the crevices of the wall, and lay basking in the white glare; and the pomegranates split and cracked with the heat, and showed their bleeding hearts. (Wilde, "Infanta" 97)

La prolepsis temática de la última línea resulta bastante elocuente. Las granadas, al abrirse y romperse, exponen sus corazones sangrantes y estipulan así un motivo temático que acabará hallando eco en el corazón roto del Enano. De igual modo, la metáfora sugiere la idea de crueldad, pero en el contexto de un jardín edénico, bello y sensual. De ese modo, la imagen "[directs] the reader to matters such as beauty, luxury, temptation and death" (Pendlebury 125); y subraya asimismo la ambivalencia del símbolo, que existe por causa de la discrepancia fundamental entre "abundance, on the one hand, and destruction on the other ... [reflecting] the challenges of accommodating pain and evil in a worldview that also encompasses passion, beauty and love" (Pendlebury 125-126). El motivo de la granada reclama una lectura que reconozca, de una parte, la exuberancia de la experiencia vital, pero sin olvidar, de otra, al mismo nivel y como parte constitutiva de la primera, las ideas de crueldad, angustia y muerte.

En este sentido, cualquier modo de lectura que pase por alto la imbricación entre el placer y la crueldad, el dolor y el sufrimiento como partes elementales de la semiótica que logra articular la experiencia vital habrá de ser, necesariamente, incompleta y equívoca. Y este no es un asunto menor, por cuanto acaso constituye el conflicto fundamental que sostiene la tragedia en miniatura protagonizada por el personaje del Enano: ${ }^{1}$

As for the Infanta, she absolutely fascinated him. He could not keep his eyes off her, and seemed to dance for her alone ... [She] took out of her hair the beautiful white rose, and partly for a jest and partly to tease the Camerera, threw it to him across the arena with her sweetest smile, he took the matter quite seriously, and pressing the flower to his rough coarse lips he put his hand upon his heart, and sank on one knee before her. (Wilde, "Infanta" 104) 
El personaje del Enano no entiende bien las reglas de los juegos de la corte, es decir, lee con inexactitud los códigos culturales que rigen el espacio de la experiencia social. La tragedia en miniatura que protagoniza se propicia en un equívoco, por medio del cual el personaje confunde el desdén y la humillación de la Infanta con una muestra de amor incondicional. ${ }^{2}$ Queda entonces atrapado el personaje en la promesa objetiva de un romance que surge por su propia incapacidad para leer el mismo código cultural en el que tal romance debería desarrollarse. Así, si "the dwarf goes down physically and psychically, becomes trapped, seduced by the erotic appeal of the Infanta, and cannot extract himself from his Hades" (Pendlebury 133) es por causa directa de la conceptualización equívoca que el Enano pone en práctica.

El narrador se toma su tiempo en afianzar el carácter inocente del Enano, entendida esta inocencia como la imposibilidad de leer los códigos culturales del horror, el sufrimiento y el dolor. Al conceptualizarse el personaje en principio como encarnación de la inocencia pastoral, sus percepciones descuellan como cándidas deconstrucciones de ritos o espacios crueles concretos. Al encontrarse, por ejemplo con la procesión que precede a una ejecución sumarísima, el Enano lee el texto cultural como "a beautiful procession winding up the long dusty road to Toledo", adornada con dulces canciones, "bright banners ..., yellow dresses painted all over with wonderful figures" (Wilde, "Infanta" 108), pero sin comprender la celebración cruel que se conforma en la actividad performativa del rito.

Tampoco entiende de manera cabal la estilizada atmósfera sombría que conforman los objetos inanimados en el interior de la corte (la rica decoración palaciega sugiere una historia de dominio que a su vez justifica la opulencia de la España barroca). Cada imagen artística de palacio muestra además una magnificencia perturbadora, que provoca en el Enano cierto sentido de lo sublime. Todas esas imágenes remiten, sin excepción, al instinto de crueldad: desde un tapiz con estampa de caza hasta unos platos decorativos grabados con la "Danza de la Muerte de Holbein" (Wilde, "Infanta" 110) pasando por los retratos de Carlos V ataviado de cazador y Felipe II recibiendo el homenaje de las colonias de Flandes (Wilde, "Infanta" 111). El deambular por los espacios de palacio suscita en el personaje un miedo perturbador que finaliza al contemplar este su propio reflejo en un espejo.

La gradación temática de la catábasis a los espacios semióticos del sufrimiento culmina en la epifanía del Enano, a causa de la cual termina comprendiendo su condición monstruosa. La circunstancia resulta demasiado cruel para poder soportarse. No se trata ahora de una intuición más o menos articulada (el sentido de lo sublime y el terror que ha sentido el Enano durante su deambular por la corte), ahora se trata de la primera ocasión en que el Enano entiende, de forma consciente y verbalizable, el horror del dolor, el sufrimiento y la crueldad (de la Infanta hacia él), resolviendo así, ya de paso, el conflicto propiciado por la lectura equívoca del símbolo de la rosa (el último acto del Enano antes de morir consiste en arrancar los pétalos de la flor). Todo esto ocurre en y a causa del espejo, cuya función trasciende la representación tradicional naturalista que atribuye Ellmann al objeto (511) y se parece más a la función que cumple el retrato de Dorian Gray, esto es, una metáfora de la representación estética que sublima y contiene la experiencia de la vida (Riquelme 626). El espejo evoca, precisamente, el código que el Enano no ha sido capaz de leer para encauzar y domar el horror intrínseco de la experiencia cultural, la cual ahora, tras el viaje iniciático que ha llevado al Enano hasta allí, se demuestra imposible de soportar para él. Resulta particularmente conmovedor a este respecto el grado de melancolía que expresa el Enano mientras evoca la inocencia recientemente perdida: "Why had they not left him", se pregunta el personaje, "where there was no mirror to tell him how loathsome he was?" (Wilde, "Infanta" 113), vinculando de manera explícita su caída a los infiernos (el dolor deletéreo de la humillación) con el conocimiento cultural implícito en la representación artística (su reflejo en el espejo). 


\section{Hermenéutica del corazón. Conclusiones}

La manifestación última de la crueldad en el relato aflora en el comentario que enuncia la infanta a la muerte del Enano: "For the future let those who come to play with me have no hearts" (Wilde, "Infanta" 114). Puede que la actitud caprichosa de la Infanta no genere muchas simpatías, como sugieren John Albert (245) y Jerusha McCormack (107). Pero entonces, siguiendo la lógica simbólica del relato, que rechaza categóricamente "the imperative to impose some form of interpretative closure" (Gillespie 14), las antipatías que pudiera suscitar la Infanta también deben ser deconstruidas, pues el relato reclama una lectura objetiva que trascienda la interpretación moral tradicional, cuestionada, por cierto, de manera flagrante desde el arranque del texto (en el símbolo, recuérdese, de la granada). Dicho de otro modo, la deconstrucción de la belleza, ligada a la muerte, al horror, la crueldad y la angustia, como texto, sugiere que la lectura de la Infanta debe solventarse en su naturaleza estética y semiótica. A su vez, implica que la lectura del relato no puede quedarse en una equivalencia directa entre el dolor y el mal. El dolor, el sufrimiento y la crueldad son, en efecto, el centro temático del relato, pero estos se dan solo por causa y en el espacio estético del estilo, en el sistema semiótico que articula el arte como ficción, la cultura como constructo canalizador de tales fenómenos. Así como Friedrich Nietzsche interpretaba que la filosofía "had become synonymous with metaphysical Truths dogmatically told to the reader in a language and systematized form that could give no account of the dynamic nature of living in the world" (Hext, "Literary Form" 702), tanto Wilde como el propio Nietzsche entienden que la moral se ha convertido en un discurso ideológico y dogmático, cerrado dialécticamente y deletéreo de la experiencia humana. Es, para ambos autores, en la experiencia estética de la cultura donde se trasciende el dolor; donde el sufrimiento adquiere, lógicamente, sentido, por causa única del espacio semiótico que lo administra.

Así las cosas, el corazón del Enano, del que tanto recela la Infanta, podría leerse en principio como símbolo de la lectura moral, cerrada (Jones 890) como tradicionalmente se ha hecho, pero precisamente porque el texto ya ha desmontado ese tipo de lectura, el corazón no puede leerse en términos morales. ${ }^{3}$ Denota esta circunstancia la dislocación entre la idea de lo bello y lo bueno, y no poder leer en estos términos implica también la incapacidad para funcionar con un tipo de código que permita soportar la crueldad como instinto constitutivo de lo humano.

La lectura moral del símbolo del corazón resulta a todas luces elusiva, y no son pocos los críticos que la han evitado al dar por sentado el significado tradicional (léase, moral) del símbolo en lugar de interpretarlo con el método, vale decir deconstructivo, de la narrativa de Wilde. Nassaar (28) no pone en duda la lectura tradicional, y apela a la interpretación del corazón como ideal moral, cristiano o pequeño burgués, lo mismo da. Otros autores, con más o menos argumentos censuran el personaje de la Infanta (Albert 244-246; McCormack 107), si no la despachan sin ambages en términos morales tradicionales (Marsh 74). Incluso Naomi Wood, aun reconociendo el proceso de resemantización que presentan ciertos símbolos y metáforas tradicionales en la obra de Wilde o la alteración significativa de las convenciones genéricas con las que trabaja el autor irlandés, sigue calificando (de manera algo acrítica, conviene señalar) al personaje de la Infanta como "monstruoso" (160). Pero Oscar Wilde, en la vena deconstructiva de su propuesta estética, "reinterpreted common myths, denied or reversed familiar metaphors" (Vicinus 85$),{ }^{4}$ de modo que resulta impropio seguir leyendo sus relatos fuera de la propuesta radicalmente estética (léase semiótica, si se quiere) de su teoría crítica, y mucho menos si se incorporan elementos de la moral tradicional sin revisión alguna, como algunos críticos todavía hacen (Wood 160; Pendlebury 132-133; Zipes 124). Véase, a modo de ejemplo, el personaje de "El alma" del pescador en "The Fisherman and His Soul", que lejos de conceptualizar un rasgo de individualidad, se revela "representative of [the] superego and 
social convention" (Zipes 124), denunciando además de paso la manipulación del significado por parte de una cultura (el discurso normativo victoriano) que hace pasar por sema de la identidad individual un constructo social privilegiado desde la ideología hegemónica. De igual modo ocurre con las iteraciones míticas de Cristo en el corpus wildeano (Valls Oyarzun 216219), las cuales simplemente no pueden leerse en el contexto de una moral tradicional cristiana:

The fact that Wilde portrayed so many Christlike protagonists in his fairy tales did not mean that he wanted to propagate the Christian way as the path toward salvation. Nor did he feel obliged to indulge himself in Christian moralizing for the sake of children as some critics have mistakenly argued ... Actually, Wilde used the figure of Christ to show the need to subvert the traditional Christian message. (Zipes 121)

Y lo mismo ocurre, naturalmente, con la lectura del corazón roto en "The Birthday of the Infanta". A este respecto, Justin Jones ya había interpretado las últimas palabras de la Infanta en el sentido de que esta no aceptaría "the hollow moral constraints that would demand sympathy for the dwarf" (891); pero con todo el acierto que acompaña a su lectura, Jones no incorpora un elemento capital, y que surge en el análisis que propone este artículo, a saber: la naturaleza semiótica implícita en la concepción estética del relato, que se desprende de la tragedia del Enano, y que se revela, porque calca, la concepción de la cultura como divinización de la crueldad propuesta por Nietzsche (una afinidad electiva entre Wilde y el filósofo alemán que no había sido explorada en profundidad hasta ahora). Carecer de corazón ("the hollow moral constraints") implica una capacidad proverbial para adaptarse al mundo como espacio hermenéutico; para aceptar y hasta abrazar la experiencia vital en toda su dimensión de belleza, placer y sufrimiento.

La resignificación de la crueldad como fundamento estético obliga a reconsiderar la función de "The Birthday of the Infanta" en el contexto global del discurso wildeano sobre la naturaleza amoral del arte o la concepción estética del mundo. No se trata solo, como defiende Justin Jones, de constatar que el relato denuncia sesgos moralistas por parte del lector victoriano al uso, porque "the spectator always sees what she wants to see in any artistic creation" $(898) ;{ }^{5}$ también debe considerarse que el relato añade al discurso de Wilde una concepción de lo estético como forma de administrar la barbarie al objeto de convertirla en civilización, transformándola y sublimándola, sin suprimirla en ningún caso. De este modo, si el arte no es más que una expresión amoral de sí mismo, tanto más constituye un acto de crueldad en sí mismo, acaso el acto de crueldad más exquisito que cabe concebir en el contex to de lo colectivo. En este sentido, "The Birthday of the Infanta" añade un matiz político a la concepción del arte por parte de Wilde, pero no porque el discurso estético pase a ser ahora una reivindicación de la dimensión política del sujeto (idea diametralmente opuesta a los principios esteticistas de Wilde), sino más bien porque la representación artística como acto de crueldad conforma el único espacio funcional en el que lo político puede acaso llegar a darse

\section{Notas}

${ }^{1}$ Llama la atención a este respecto que la crítica especializada en la literatura infantil de Wilde apenas si haya comentado el origen de la peripecia del Enano. Nassaar (1974), Albert (1991), McCormack (1996), Wood (2002), Pendlebury (2011) o Jones (2011), por ejemplo, pasan de puntillas por el incidente que propicia su viaje iniciático ya que prefieren detenerse en la lectura moral que subyace a su destino trágico. No yerran del todo en su estrategia, es verdad, pero la ponen en práctica de forma limitada, pues al no tomar bien en consideración el origen del conflicto fundamental del Enano, no terminan tampoco de contextualizar las lecturas que proponen a ese respecto. Dicho de otro modo, ninguno de ellos acaba de leer el cuento como lo que es, una tragedia, a pesar de que sí consideren el pathos fundamental que caracteriza al personaje del Enano. 


\begin{abstract}
${ }^{2}$ Resulta bastante elocuente a este respecto que el Enano confunda el significado de una rosa blanca con el sentido simbólico tradicional de la rosa roja.

${ }^{3}$ Esta deconstrucción del símbolo "corazón" como emblema de la moral tradicional se da de algún modo también en The Picture of Dorian Gray (1891). El corazón descuella al final de la novela en el mismo sentido en que lo hace en "The Birhday of the Infanta". Dorian Gray apuñala el retrato justo en el corazón (Wilde, Dorian Gray 246) atacando así el espacio simbólico que define el retrato, desde una perspectiva victoriana, como conciencia moral del personaje. Al partirse el corazón, el arte se separa de la lectura ética y recobra su estructura autoreferrencial, trascendiendo al mismo tiempo el prejuicio moral que supone ver el arte a través de un código tradicional ajeno a la propia norma estética. Así, el significado del corazón como "hollow moral constraint" (Jones 891) que tan bien funciona en "The Birthday of the Infanta" casa también de forma adecuada con la lectura deconstruida del símbolo que se sugiere la novela.

${ }^{4}$ Vicinus aplica este comentario a un grupo indeterminado de "escritores homosexuales" (85). El grupo incluye, lógicamente, al propio Wilde.

${ }^{5}$ Esta afirmación de Justin Jones es parte de un comentario sobre tres de los relatos contenidos en A House of Pomegranates: "The Young King", "The Star-Child" además del propio "The Birthday of the Infanta".
\end{abstract}

\title{
Works cited
}

Albert, John. "The Christ of Oscar Wilde". Critical Essays on Oscar Wilde. Ed. Regenia Gagnier. Toronto: Maxwell MacMillan, 1991. 241-257.

Allen, James Sloan. "Nietzsche and Wilde an Ethics of Style". Sewanee Review 114.3 (2006): 386-404.

Bridgewater, Patrick. Anglo-German Interactions in the Literature of the 1890s. London: Routledge, 1999.

Brown, Julia Prewitt. Cosmopolitan Criticism: Oscar Wilde's Philosophy of Art. Charlottesville and London: University Press of Virginia, 1997.

Deleuze, Gilles. Nietzsche y la filosofía. Trad. C. Artal. Barcelona: Anagrama, 2002 (1968).

Ellmann, Richard. Oscar Wilde. Nueva York: Vintage, 2000 (1987).

Freud, Sigmund. El malestar en la cultura. Trad. R. Rey Ardid. Madrid: Alianza, 2003 (1930).

Gillespie, Michael Patrick. Oscar Wilde and the Poetics of Ambiguity. Gainesville: University Press of Florida, 1996.

Hext, Kate. "Oscar Wilde and Friedrich Nietzsche: 'Rebels in the name of beauty". Victoriographies 1. 2 (2011): 202-220.

. "Literary Form and Philosophical Thought in Nineteenth-Century Britain". Literature Compass 9.11 (2012): 695-707.

Jones, Justin T. "Morality's Ugly Implications in Oscar Wilde's Fairy Tale”. Studies in English Literature 1500-1900 51.4 (2011): 883-903.

Mann, Thomas. "Wilde and Nietzsche". Oscar Wilde: A Collection of Critical Essays. Ed. Richard Ellmann. New Jersey: Prentice Hall, 1969. 169-171.

Marsh, Sarah. "Twice Upon a Time: The Importance of Rereading "The Devoted Friend". Children's Literature 36 (2008): 72-87.

McCormack, Jerusha. "Wilde's Fiction(s)". The Cambridge Companion to Oscar Wilde. Ed. P. Raby. Cambridge: CUP, 1996. 96-117.

Nassaar, Christopher. S. Into the Demon Universe: A Literary Exploration of Oscar Wilde. New Haven and London: Yale University Press, 1974.

Nietzsche, Friedrich. "El Anticristo". Obras completas: obras de madurez II, vol. 4. Ed. D. Sánchez Meca. Madrid: Tecnos, 2016. 694-773.

. "La genealogía de la moral". Obras completas: obras de madurez II, vol. 4. Ed. D. Sánchez Meca. Madrid: Tecnos, 2016. 440-562.

. "Más allá del bien y del mal". Obras completas: obras de madurez II, vol. 4, Ed. D. Sánchez Meca. Madrid: Tecnos, 2016. 282-439. 
Pendlebury, Kate. "The Building of A House of Pomegranates". Marvels \& Tales: Journal of Fairy-Tale Studies 25.1 (2011): 124-141.

Riquelme, John Paul. “Oscar Wilde's Aesthetic Gothic: Walter Pater, Dark Enlightenment and The Picture of Dorian Gray". Modern Fiction Studies 46.3 (2000): 609-631.

Safranski, Rüdiger. Nietzsche: Biografía de su pensamiento. Trad. R. Gabás. Barcelona: Tusquets, 2000.

Valls Oyarzun, Eduardo. Dueños del tiempo y del espanto: genealogía nietzscheana de la responsabilidad en la narrativa victoriana. Madrid: Escolar y Mayo, 2017.

Vicinus, Martha. "The Adolescent Boy: Fin-de-Siècle Femme Fatale?" Victorian Sexual Dissidence. Ed. R. Dellamora. Chicago and London: The University of Chicago Press, 1999. 83-107.

Wilde, Oscar. "The Birthday of the Infanta". Complete Short Fiction. New York: Penguin. 1994. 97-114.

. "The Critic as Artist". De Profundis and Other Writings. New York: Penguin. 1986. 135-211.

—. "The Fisherman and His Soul". Complete Short Fiction. New York: Penguin, 1994. 115-148.

. The Picture of Dorian Gray. New York: Penguin, 1992.

Wood, Naomi. "Creating the Sensual Child: Paterian Aesthetics, Pederasty, and Oscar Wilde's Fairy Tales”. Marvels \& Tales: Journal of Fairy-Tale Studies 16.2 (2002): 156-170.

Zipes, Jack. Fairy Tales and the Art of Subversion. The Classical Genre for Children and the Process of Civilization. London: Routledge, 2012.

Received: 28 October $2019 \quad$ Revised version accepted: 21 January 2020

Eduardo Valls Oyarzun is Associate Professor of English and American literature at Complutense University of Madrid. He specializes in nineteenth century literature, and has published extensively on Oscar Wilde, George Bernard Shaw, Joseph Conrad and Lewis Carroll. His most recent books comprise a critical edition of George Bernard Shaw's The Perfect Wagnerite (Madrid, Alianza Editorial, 2011) and the monograph Dueños del tiempo y del espanto genealogía nietzscheana de la responsabilidad en la narrative victoriana (Madrid, Escolar y Mayo, 2017) (Masters of Time and Horror: A Nietzschean Genealogy of Responsibility in Victorian fiction).

evallsoyarzun@filol.ucm.es 